\title{
Controlling the Optical Properties of Intersecting Waveguides: Fractionally Doping the Intersection Region
}

\author{
Douglas M. Gill, Leon McCaughan, Member, IEEE, and Niraj Agrawal
}

\begin{abstract}
Modification of the refractive index of the region common to intersecting waveguides (fractional doping) provides a mechanism, independent of intersection angle, for controlling the optical coupling characteristics of this geometry. We derive a pair of expressions based on a multiple scattering analysis of intersecting waveguides which predict the coupling behavior as a function of intersection angle and fractional doping for both polarizations. The expressions are in good agreement with experimental data from several different laboratories. The conditions for polarization-independent coupling and polarization splitting are demonstrated.
\end{abstract}

\section{INTRODUCTION}

TNTERSECTING waveguides are a fundamental guided wave Lelement for integrated optics. As a passive element, this geometry makes possible high-density electrooptic switch arrays [1], [2], passive polarization splitters [3], [4], and offers the possibility of wavelength multiplexing. Optical switches have been fabricated using intersecting waveguides in an electrooptic substrate [5]. In the design of intersecting guides, both the coupling strength between the guides and their insertion loss are functions of the optical confinement (as characterized by the $V$-parameter) as well as the intersection angle [6]-[8]. This interdependence limits design flexibility. We demonstrate theoretically and experimentally that a third processing parameter, the refractive index at the intersection of the two waveguides, controls the guided-guided field coupling as effectively as the intersection angle. As will be discussed below, fractional doping also effects the radiative loss of this geometry. Selective doping of the intersection region therefore introduces a new processing degree of freedom, apart from intersection angle, in the design and optimization of integrated optics employing intersecting waveguides.

A recently developed theory based on a multiple scattering analysis of TE polarized light in intersecting symmetric slab waveguides correctly predicts the coupling and radiative loss behaviors of TM polarized light in $\mathrm{Ti}: \mathrm{LiNbO}_{3}$ channel waveguides as a function of intersection angle [7]-[9]. Intersecting guides are typically fabricated with either a constant film index throughout the guiding region (i.e., the single- $\Delta n$ geometry) or with the film index of one guide superimposed on the other (the $2-\Delta n$ geometry). As will be shown, the theory predicts and

Manuscript received May 14, 1990; revised November 14, 1990

D. M. Gill is with the Materials Science Program, University of Wisconsin, Madison, WI 53706

L. McCaughan is with the Department of Electrical and Computer En gineering, University of Wisconsin, Madison, WI 53706.

N. Agrawal is with the IBM Research Division, Almaden Research Center, San Jose, CA 95120

IEEE Log Number 9143418. experiments corroborate that a fractional change in the refractive index in the intersection region will alter the coupling characteristics as effectively as changing the intersection angle.

\section{THEORY}

The multiple scattering analysis [7], [8] treats one planar waveguide of an intersecting pair as an extended scattering element of the other. Light in each guide is successively scattered into the guided and radiation fields of the other guide. The process is repeated self-consistently and the electric field associated with the $i$ th waveguide is expressed as a series of scattering interactions,

$$
E_{i}(y, z)=\sum_{n=0} E_{i}^{(n)}(y, z)
$$

where $i=1,2$ represents the respective waveguides and $n$ is the order of the scattering term. The $r$ th order scattered fields $E_{x, 1}^{(n)}$ and $E_{x, 2}^{(n)}$ satisfy

$$
\begin{aligned}
& {\left[\nabla_{t}^{2}+\omega^{2} \mu\left(\epsilon_{s}+\epsilon_{2}\right)\right] E_{x, 2}^{(n)}=-\omega^{2} \mu\left(\epsilon_{2}+l\right) E_{x, 1}^{(n-1)}} \\
& {\left[\nabla_{t}^{2}+\omega^{2} \mu\left(\epsilon_{s}+\epsilon_{1}\right)\right] E_{x, 1}^{(n)}=-\omega^{2} \mu\left(\epsilon_{1}+l\right) E_{x, 2}^{(n)}}
\end{aligned}
$$

where $\epsilon_{i}$ is the dielectric permittivity of the $i$ th waveguide and $l$ is the permittivity associated with the intersection region (Fig. 1).

The solution to (2) for the TE polarization can be expressed in terms of the Green's function $G_{i}\left(y, z, y^{\prime}, z^{\prime}\right)$ for the individual waveguides:

$$
\begin{aligned}
E_{x, 2}^{(n)}(y, z)= & i \omega / 4 \int_{-\infty}^{+\infty} \int_{-\infty}^{+\infty}\left[\epsilon_{2}\left(y^{\prime} z^{\prime}\right)+l\left(y^{\prime}, z^{\prime}\right)\right] \\
& \cdot E_{x, 1}^{(n-1)}\left(y^{\prime}, z^{\prime}\right) G_{2}\left(y, z ; y^{\prime}, z^{\prime}\right) d y^{\prime} d z^{\prime} \\
E_{x, 1}^{(n)}(y, z)= & i \omega / 4 \int_{-\infty}^{+\infty} \int_{-\infty}^{+\infty}\left[\epsilon_{1}\left(y^{\prime} z^{\prime}\right)+l\left(y^{\prime}, z^{\prime}\right)\right] \\
& \cdot E_{x, 2}^{(n)}\left(y^{\prime}, z^{\prime}\right) G_{1}\left(y, z ; y^{\prime}, z^{\prime}\right) d y^{\prime} d z^{\prime}
\end{aligned}
$$

where the Green's function can be written as a sum of guided and radiation field components,

$$
G_{i}=G_{i}^{(g)}+G_{i}^{(r)} .
$$

For conditions in which the radiation losses can be neglected $\left[G^{(r)}=0\right.$ ], the optical power coupled between intersecting waveguides using (1) and (3) with $n \rightarrow \infty$, becomes [7]

$$
P_{x}=\sin ^{2} K
$$




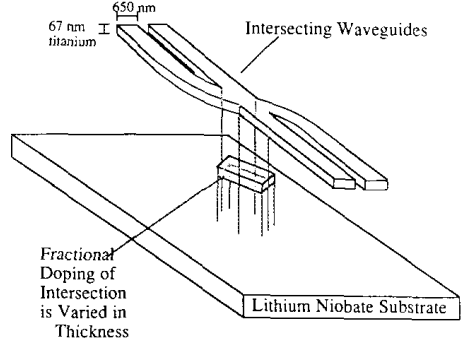

Fig. 1. Schematic diagram of $\mathrm{Ti}: \mathrm{LiNbO}_{3}$ fractionally-doped intersecting waveguide fabrication. The Ti thickness of the intersection region relative to the waveguide arms was varied from $\Delta \tau / \tau_{0}=0$ to $\Delta \tau / \tau_{0}=1$.

where

$$
K=K^{\mathrm{ev}}+K^{\mathrm{fd}}
$$

Here $K^{\mathrm{ev}}$ is the contribution to the coupling coefficient from the overlap of the evanescent field of one waveguide with the other guide. Physically, $K^{\mathrm{ev}}$ corresponds to the single- $\Delta n$ geometry (i.e., the guiding index in the intersection is the same as that in the waveguide arms). $K^{\mathrm{fd}}$ is the contribution to the coupling coefficient from the additional doping of the intersection region above that of the individual waveguides. As the intersection region is fractionally doped, the $K^{\text {ev }}$ term remains constant while the $K^{\mathrm{fd}}$ term monotonically increases. The contribution from the fractional dopant in the intersection region is given by

$$
\begin{aligned}
K^{\mathrm{fd}}= & \frac{\omega^{2} \mu \Delta \epsilon_{\mathrm{fd}}}{2 \beta\left(a+\frac{1}{\gamma}\right) \sin \theta} \times\left[\frac{\sin \left[\left(\alpha-\beta \tan \frac{\theta}{2}\right) a\right]}{\left(\alpha-\beta \tan \frac{\theta}{2}\right)}\right. \\
& \left.+\frac{\sin \left[\left(\alpha+\beta \tan \frac{\theta}{2}\right) a\right]}{\left(\alpha+\beta \tan \frac{\theta}{2}\right)}\right]^{2}
\end{aligned}
$$

where $\beta$ and $2 a$ are the propagation constant and guiding film thickness, respectively, of individual waveguides. Here $\Delta \epsilon_{\mathrm{fd}}$ is the fractional doping permittivity (above that of the guiding layer). The constants $\alpha$ and $\gamma$ are determined from the dispersion relation

$$
\gamma=\alpha \tan (\alpha a)
$$

where $\alpha$ and $\gamma$ are the magnitudes of the transverse components of the wave vectors in the film and substrate, respectively.

The multiple scattering analysis is derived from a treatment of TE polarized light in a pair of intersecting slab waveguides. Intersecting channel waveguides with light polarized normal to the substrate surface (i.e., TM polarization) most closely corresponds to this model. In the following calculations and measurements we treat Ti-diffused channel waveguides in $z$-cut $\mathrm{LiNbO}_{3}$. For TM-polarized light the refractive index varies linearly with $\mathrm{Ti}$ concentration [10] and is therefore taken to be proportional to the $\mathrm{Ti}$ thickness before diffusion. In the intersection region the $\mathrm{Ti}$ thickness is $\tau_{0}+\Delta \tau$, where $\tau_{0}$ is the $\mathrm{T}$ thickness of the waveguide arms and $\Delta \tau$ is the thickness of the additional doping in the intersection (see Fig. 1). The change in permittivity in the intersection region $\Delta \epsilon_{\mathrm{fd}}$ above that in a waveguide $\epsilon_{f}$ is therefore related to the fractional doping thickness of $\mathrm{Ti}, \Delta \tau / \tau_{0}$ by

$$
\Delta \epsilon_{\mathrm{fd}} \cong\left(\epsilon_{f}-\epsilon_{s}\right) \Delta \tau / \tau_{0}
$$

where $\epsilon_{f}$ and $\epsilon_{s}$ are the permittivities of the guiding film and substrate, respectively, of the slab model waveguide. For the special case of a $2 \Delta n$ structure $\Delta \epsilon_{\mathrm{fd}}=\left(\epsilon_{f}-\epsilon_{s}\right)$. Referring to (2)

$$
l=\left(\epsilon_{f}-\epsilon_{s}\right)\left(\Delta \tau / \tau_{0}-1\right)
$$

\section{EXPERIMENT}

Fractionally-doped intersecting waveguides were fabricated in $z$-cut $y$-propagating $\mathrm{LiNbO}_{3}$ in two steps: intersecting waveguides were photolithographically patterned in $\mathrm{Ti}\left(\tau_{0} \sim 67.5\right.$ $\mathrm{nm}$ thick by $w=6.5 \mu \mathrm{m}$ wide) over the fractional doping rectangle of Ti with thickness $\Delta \tau$ (Fig. 1). The rectangle is $6.5 \mu \mathrm{m}$ wide and spans the length between intersecting waveguide vertices. Thermal diffusion of the Ti into the $\mathrm{LiNbO}_{3}$ substrate under wet oxygen at $1050^{\circ} \mathrm{C}$ for $6 \mathrm{~h}$ produces low loss $(\leqslant 0.2$ $\mathrm{dB} / \mathrm{cm}$ ) strongly-confining channel waveguides [11]. Measurements were made by lens-coupling light of $\lambda=1.3 \mu \mathrm{m}$ wavelength (polarized normal to the $\mathrm{LiNbO}_{3}$ surface for the TM polarization and parallel to the substrate surface for the TE polarization). Waveguide coupling was measured as a function of the fractional doping parameter $\Delta \tau / \tau_{0}$ over a range from (1 $\left.+\Delta \tau / \tau_{0}\right)=1$ (single- $\Delta n$ geometry) to a value twice that in the waveguide arms, $\left(1+\Delta \tau / \tau_{0}\right)=2(2-\Delta n$ geometry).

\section{Discussion}

Waveguide-waveguide coupling for the TM polarization was measured and calculated (using the multiple scattering theory) as a function of fractional doping of the intersection region for three intersecting angles, $0.5^{\circ}, 1.0^{\circ}$, and $1.5^{\circ}$ [Fig. 2 (a)-(c)]. Coupling ratios are a strong function of the normalized film thickness ( $V$-parameter) for intersecting waveguides,

$$
V=\frac{4 \pi a}{\lambda} \sqrt{n_{f}^{2}-n_{s}^{2}}
$$

where $2 a$ is the waveguide width. A value of $V / \pi=0.82 \pm$ 0.02 produces an optimum agreement between the multiple scattering calculations and coupling measurements made as a function of fractional doping (Fig. 2). This value is consistent with well-confined single-mode waveguides $(V / \pi \leqslant 1)$ and was used for all subsequent coupling calculations. (The exact choice of the values of film index, $n_{f}=2.204$, cladding index, $n_{c}=$ 2.200 , and film width, $2 a=4 \mu \mathrm{m}$, is of less importance to the theoretical results than the value of $V$ itself). The data (Fig. 2) are consistent with (5)-(7) and (9): the fraction of light coupled goes as $\sin ^{2} K$ and $K$ varies linearly with fractional doping, $\Delta \tau / \tau_{0}$.

The fraction of light coupled between waveguides measured as a function of intersection angle is plotted for both polarizations for the single- $\Delta n^{12}$ and $2 \Delta n^{13}$ (i.e., $\Delta \tau / \tau_{0}=0$ and 1) geometries in Figs. 3 and 4, respectively. Both polarizations in both geometries have the form of (5) (which has been derived only for the TM mode). Note that the coupling coefficient $K$ decreases with increasing intersection angle in all cases. This last fact is more clearly seen by plotting the coupling coefficient [using (5)] as a function of intersection angle (Figs. 5 and 6). The multiple scattering calculations for the TM polarization (dashed lines, Fig. 5) are in excellent agreement with data. The 


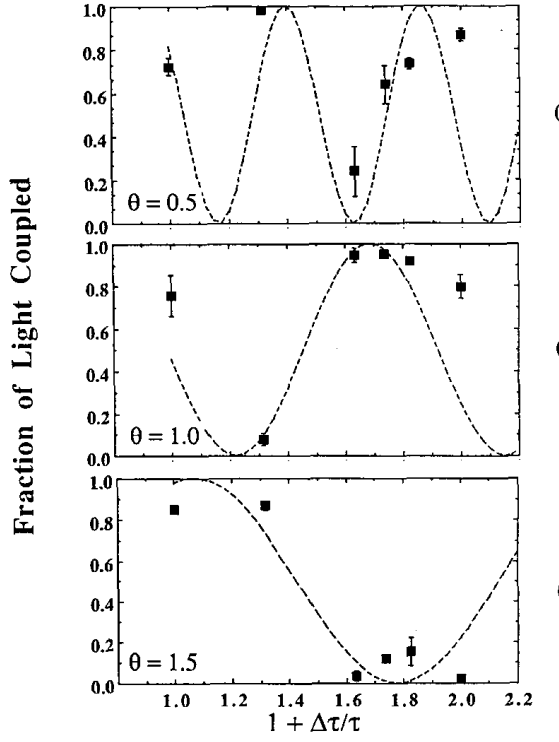

(a)

(b)

Fig. 2. Measured fraction of light intensity (TM polarization) coupled between intersecting waveguides as a function of fractional doping. Error bars are based on a Gaussian distribution of measurements on four nominally identical structures. Multiple scattering calculation results are shown as a dashed line. Intersection angles (a) $0.5^{\circ}$, (b) $1.0^{\circ}$, and (c) $1.5^{\circ}$

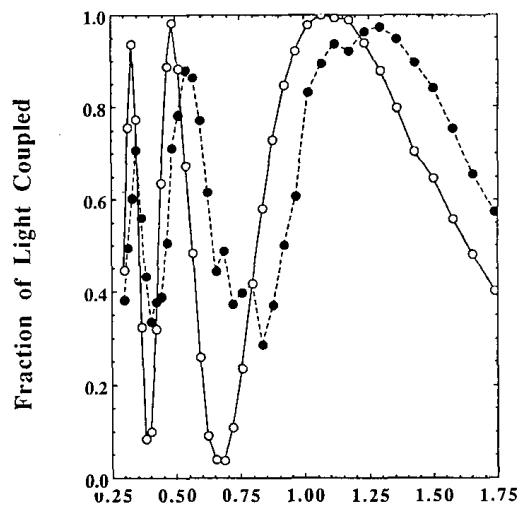

Intersection Angle (Degrees)

Fig. 3. Light intensity coupled between single- $\Delta n$ intersecting waveguides (i.e., guiding index is everywhere the same) measured as a function of intersection angle for the TE $(O)$ and TM $(\bullet)$ polarization [12]. Data points are connected by a solid line.

coupling coefficients can be expressed in a more simple form than (7) by taking advantage of the fact that $K^{\mathrm{ev}}$ is independent of fractional doping and $K^{\mathrm{fd}}$ depends linearly on $\Delta \tau / \tau_{0}$ [see (7)]. From (6) and (7) the coupling coefficients for the single$\Delta n$ and $2 \Delta n$ geometries become $K_{1 \Delta n}=K^{\mathrm{ev}}$ and $K_{2 \Delta n}=K^{\mathrm{ev}}$ $+K^{\mathrm{fd}}\left(\Delta \tau / \tau_{0}=1\right)$. The coupling coefficient data for the TM polarization (Fig. 5) was therefore fit to the form

$$
K_{\mathrm{TM}}=K^{\mathrm{ev}}+K^{\mathrm{fd}}-2.0 \times \theta^{-1.33}+3.2\left(\Delta \tau / \tau_{0}\right) \theta^{-1.02}
$$

where $\theta$ is expressed in degrees. The multiple scattering analysis for the TE polarization in channel waveguides has not yet

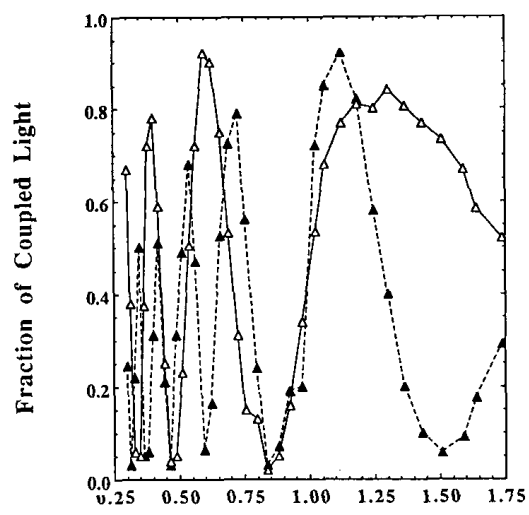

Intersection Angle (Degrees)

Fig. 4. Light intensity coupled between $2-\Delta n$ intersecting waveguides (i.e. index in intersection region is twice that in the guiding arms) measured as a function of intersection angle for the TE $(\Delta)$ and TM $(\Delta)$ polarizations. Data points are connected by a solid line.

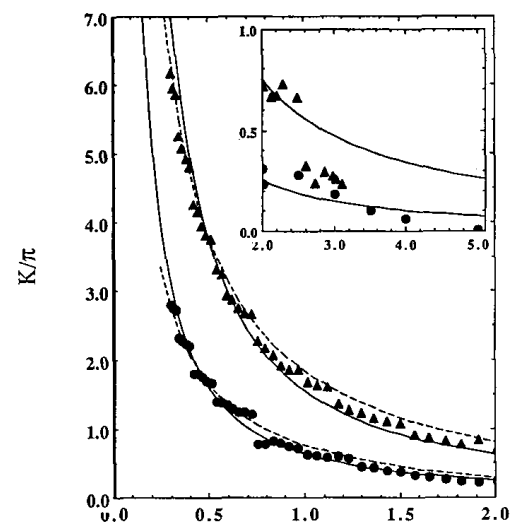

Intersection Angle (Degrees)

Fig. 5. Plot of the TM polarization coupling coefficient derived from coupling data (Figs. 3 and 4 ) versus intersection angle for the single- $\Delta n(\bullet)^{12}$ and $2 \Delta n(\Delta)^{i 3}$ cases. Data from [6] $(\bullet)$ and [13] $(\Delta)$ are shown in the inset. Dashed lines are the coupling coefficients versus $\theta$ predicted from the multiple scattering analysis. Solid lines are plots of (12).

been derived. As an approximation, we assume a coupling coefficient of the form of (12) and approximate the TE polarization data (Fig. 6) by

$K_{\mathrm{TE}}=K_{\mathrm{ev}}+K_{\mathrm{fd}} \sim 1.6 \times \theta^{-1.45}+0.44\left(\Delta \tau / \tau_{0}\right) \theta^{-1.66}$.

Equations (12) and (13) are plotted (solid lines) with the coupling data in Figs. 5 and 6 . Note that the figures contain data from four sources ([6], [12], [13], and this work). Note also that these approximations are good fits to the entire range of data $\left(\theta \leq 5^{\circ}\right.$ for the single- $\Delta n$ and $\theta \leq 3^{\circ}$ for the $2 \Delta n$ configurations).

Equally significant, (12) and (13) can be used to represent the coupling data for dopings of the intersection region other than single- $\Delta n\left(\Delta \tau / \tau_{0}=0\right)$ and $2 \Delta n\left(\Delta \tau / \tau_{0}=1\right)$ configurations. The coupling data of fractionally doped intersecting 


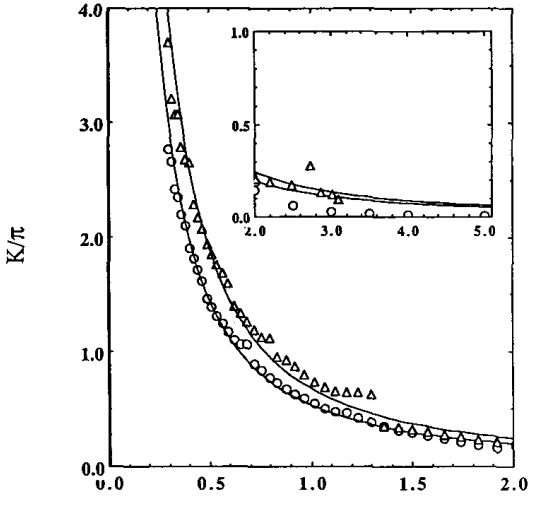

Intersection Angle (Degrees)

Fig. 6. Plot of the TE polarization coupling coefficient derived from coupling data (Figs. 3 and 4 ) versus intersection angle for the single- $\Delta n(O)^{12}$ and $2 \Delta n(\Delta)^{13}$ cases. Data from [6] $(O)$ and $[13](\Delta)$ are shown in the inset. Solid lines are plots of (13).

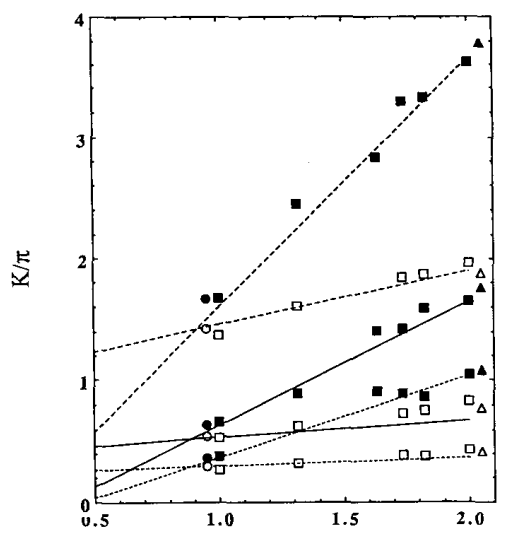

$1+\Delta \tau / \tau$

Fig. 7. Plot of the TE ( $\square$ ) and TM ( $\square$ ) coupling coefficient data versus fractional doping for intersection angles $\theta=0.5^{\circ}, 1.0^{\circ}$, and $1.5^{\circ}$. Data from [12] $(\bullet, O)$ and [13] $(\triangle, \Delta)$ are included and offset from their proper positions for clarity. Plots of (12) and (13) are included for $\theta=$ $0.5^{\circ}$ (dotted line), $1.0^{\circ}$ (solid line), and $1.5^{\circ}$ (dashed line).

guides (Fig. 2), and the data from [12] and [13], have been replotted in terms of $K$ as a function of fractional doping in Fig. 7. Also included in Fig. 7 is the coupling data for the TE polarization. Equations (12) and (13) (solid lines) are seen to be an excellent fit to all of the data. A few points on this figure are worth noting. In general, $\partial K / \partial \tau$ and $\partial K / \partial \theta$ are unequal (see Figs. 5, 6, and 7) for the two polarizations. It should therefore always be possible [using either the multiple scattering analysis or an approximation of the form (12) and (13)] to choose a fractional doping $\Delta \tau / \tau_{0}$ which produces polarization-independent coupling of arbitrary splitting ratio for some intersection angle $\theta$. Note that it is not necessary that the coupling coefficients be equal, just that $K_{\mathrm{TE}(\mathrm{TM})}=m \pi \pm K_{\mathrm{TM}(\mathrm{TE})}, m=0,1,2 \cdots$. A particularly striking example of this is the value $\Delta \tau / \tau_{0}=-0.1$, for which the TE and TM coupling coefficients are approxi- mately equal at all three of the intersection angles investigated. This suggests that by making the intersecting region with $\sim 90 \%$ $\left(\Delta \tau / \tau_{0}=-0.1\right)$ of the doping of the waveguide arms with $0.5^{\circ}$ $<\theta<1.5^{\circ}$, robust polarization-independent couplers can be made. Note that insertion losses are predicted to increase $\sim 1-$ $3 \mathrm{~dB}$ (depending on intersection angle) for this specific intersecting configuration [14]. Over this range $0.1<K / \pi<1.2$, which encompasses one complete coupling cycle. Conversely, Fig. 7 shows that choosing $\Delta \tau / \tau_{0}-1.0$ and $\theta=0.5^{\circ}$ or $1.5^{\circ}$ produces polarization splitting. We obtained a TE to TM contrast ratio of $\sim 20 \mathrm{~dB}$ for these intersection geometries.

As we have shown elsewhere [14], fractional doping can increase or decrease the radiative loss from intersecting waveguides. In the example above a fractional doping of $0.4<$ $\Delta \tau / \tau_{0}<0.8$ produces $-2 \mathrm{~dB}$ reduction in loss for the TE polarization and $\sim 3 \mathrm{~dB}$ reduction in loss for the TM polarization below that of single $\Delta n$ intersecting guides [14]. This range of $\Delta \tau / \tau_{0}$ is sufficiently large that low loss intersections with arbitrary coupling ratios for the two polarizations can be obtained by properly choosing the intersection angle and fractional doping.

\section{CONCLUSION}

It has been demonstrated that the guided-guided TM field coupling between intersecting waveguides can be explained by the multiple scattering analysis. We have derived a simplified expression for the coupling coefficient of both the TE and TM polarizations using the multiple scattering results and coupling data. These expressions have been shown to accurately predict the coupling behavior for well-confined $\mathrm{Ti}: \mathrm{LiNbO}_{3}$ channel waveguides with arbitrary doping levels of the intersection region. We have also shown that fractional doping is as important a design parameter as the intersection angle, both of which can be used to "tune" the coupling characteristics of both polarizations in a predictable manner. In particular, polarization-independent coupling and polarization splitting at several intersection angles is possible with the appropriate fractional doping. Finally, with regard to integrated optic fabrication and design, we note that design errors at the mask level can be compensated during processing by incorporating fractional doping as part of the fabrication process. Taken together, these results demonstrate a new predictable processing parameter, fractional doping, for the development of intersecting waveguide structures.

\section{ACKNOWLEDGEMENT}

The authors would like to acknowledge the help of J. Watson of AT\&T Bell Laboratories, Breinigsville, PA, for assistance in fabricating some of the photolithographic masks.

\section{REFERENCES}

[1] J. J. Veselka, T. O. Murphy, D. A. Herr, J. E. Watson, M. A. Milbrodt, K. Bahadori, M. F. Dautartas, C. T. Kemmerer, D. T. Moser, A. W. Schelling, "Low-voltage low-crosstalk $8 \times 8$ Ti : $\mathrm{LiNbO}_{3}$ switch for a time multiplexed switching system," in Proc. Opt. Fiber Conf., Houston, TX, 1989.

[2] G. A. Bogert, " $4 \times 4 \mathrm{Ti}: \mathrm{LiNbO}_{3}$ switch array with full broadcast capability," presented at Top. Meet. Photon. Switching, Incline Village, NV, Mar. 1987, paper ThD3-1.

[3] H. Nakajima, T. Horimatsu, M. Seino, and I. Sawaki, "Crosstalk characteristics of $\mathrm{Ti}: \mathrm{LiNbO}_{3}$ intersectıng waveguides and their applications as TE/TM mode splitters," IEEE Trans. Microwave Theory Tech., vol. MTT-30, p. 617, 1982. 
[4] A. Neyer, "Low-crosstalk passive polarization splitters using Ti : $\mathrm{LiNbO}_{3}$ waveguide crossings,' Appl. Phys. Lett., vol. 55, p. 927, 1989.

[5] - "Electro-optic X-Switch using single-mode $\mathrm{Ti}: \mathrm{LiNbO}_{3}$ channel waveguides," Electron. Lett., vol. 19, p. 553, 1983.

[6] G. A. Bogert, "Ti: LiNbO3 Intersecting waveguides," Electron. Lett., vol. 23, pp. 72-73, 1987.

[7] N. Agrawal, L. McCaughan, and S. R. Seshadri, "A multiple scattering analysis of intersecting waveguides," J. Appl. Phys., vol. 62 , p. $2187,1987$.

[8] N. Agrawal and L. McCaughan, "Radiation losses in intersecting optical waveguides," J. Appl. Phys., vol. 65, p. 4509, 1989.

[9] - , "Low-loss Ti: LiNbO3 intersecting waveguides," Appl. Phys. Lett., vol. 54, p. 1669, 1989.

[10] M. Minakata, S. Saito, M. Shibata, and S. Miyazawa, "Precise determination of refractive index changes in Ti-diffused $\mathrm{LiNbO}$ optical waveguides," J. Appl. Phys., vol. 49, p. 4677, 1978.

[11] L. McCaughan and E. J. Murphy, "Influence of temperature and initial Ti dimensions on fiber-TiLiNbO insertion loss," IEEEJ. Quantum Electron., vol. QE-19, p. 131, 1983.

[12] N. Agrawal, "Novel physical effects in intersecting optical waveguides," Ph.D. dissertation, 1988.

[13] E. E. Bergmann, L. McCaughan, and J. E. Watson, "Coupling of intersecting $\mathrm{Ti}: \mathrm{LiNbO}_{3}$ diffused waveguides," Appl. Opt. vol. 23 , p. $3000,1984$.

[14] D. Gill, N. Agrawal, and L. McCaughan, "Reducing radiative loss in intersecting waveguides by fractionally doping the intersection region," IEEE Photon. Technol. Lett., to be published.

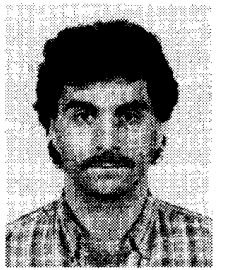

Douglas M. Gill was born in Syracuse, NY on September 5, 1962. He received the B.S degree in physics from the State University of New York, College at Buffalo, in 1987, and the M.S. degree in materials science from the University of Wisconsin, Madison, in 1989.

Currently, he is engaged in Ph.D. research with the University of Wisconsin. His current research interests include the study of integrated optics with localized gain.

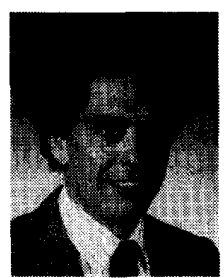

Leon McCaughan (M'84) received the M.S and $\mathrm{Ph} . \mathrm{D}$. degrees in physics and biophysics, respectively, from the University of Michigan. Ann Arbor.

From 1979 to 1980 , he held a postdoctoral appointment with Brookhaven National Labo ratory. From 1980 to 1984 , he was with Bell Laboratories, where he was engaged in the research and development of integrated optic devices. $\mathrm{He}$ is currently an Associate Professor with the Department of Electrical and Com puter Engineer, University of Wisconsin, Madison. His research in cludes integrated optics, nonlinear optics, and fiber optics.

Niraj Agrawal, photograph and biography not available at the time of publication. 